\title{
Práticas terapêuticas com plantas medicinais para o tratamento do Diabetes Mellitus
}

\author{
Therapeutic practices with medicinal plants for the treatment of Diabetes Mellitus \\ Prácticas terapéuticas con plantas medicinales para el tratamiento de la Diabetes Mellitus
}

Recebido: 29/07/2021 | Revisado: 07/08/2021 | Aceito: 13/08/2021 | Publicado: 16/08/2021

\author{
Bárbara Mendes Guimarães \\ ORCID: https://orcid.org/0000-0001-8607-6417 \\ Universidade Federal dos Vales Jequitinhonha e Mucuri, Brasil \\ E-mail: barbara.guimaraes@ufvjm.edu.br \\ Karla Antunes Ramos \\ ORCID: https://orcid.org/0000-0003-2590-2724 \\ Universidade Federal dos Vales Jequitinhonha e Mucuri, Brasil \\ E-mail: karla.ramos@ufvjm.edu.br \\ Marcio Coutinho de Souza \\ ORCID: https://orcid.org/0000-0002-4238-1572 \\ Universidade Federal dos Vales Jequitinhonha e Mucuri, Brasil \\ E-mail: márcio.souza@ufvm.edu.br \\ Mauro Lúcio Franco \\ ORCID: https://orcid.org/0000-0003-2114-4399 \\ Universidade Federal dos Vales Jequitinhonha e Mucuri, Brasil \\ E-mail: mlfranco@ufvjm.edu.br \\ Caio César de Souza Alves \\ ORCID: https://orcid.org/0000-0001-9765-8527 \\ Universidade Federal dos Vales Jequitinhonha e Mucuri, Brasil \\ E-mail: caio.alves@ufvjm.edu.br \\ Alessandra de Paula Carli \\ ORCID: https://orcid.org/0000-0002-7956-8947 \\ Universidade Federal dos Vales Jequitinhonha e Mucuri, Brasil \\ E-mail: alessandrapcarli@hotmail.com \\ Jandesson Mendes Coqueiro \\ ORCID: https://orcid.org/0000-0002-5321-5174 \\ Universidade Federal do Espírito Santo, Brasil \\ E-mail: jandesson.mc@gmail.com
}

\begin{abstract}
Resumo
Os registros de práticas terapêuticas com uso de plantas medicinais encontram-se desde a prevenção, tratamento e cura de doenças sendo de grande relevância para a história da humanidade. Destarte, esse artigo tem por objetivo identificar as plantas medicinais utilizadas na prática terapêutica para o tratamento do Diabetes Mellitus. Justifica-se essa pesquisa também devido ao grande número de pessoas acometidas pela doença. Para tanto, foi realizada uma pesquisa bibliográfica, exploratória e qualitativa. Dessa forma para compor essa pesquisa foi realizada a busca em bases de dados (Google Scholar), (PubMed) e (Scielo) utilizando descritores em Ciências da Saúde (DeCS): diabetes mellitus" e "plantas medicinais" ou seus equivalentes no inglês e espanhol, sendo selecionadas as referências que compreendem o período de 1993 a 2021. Discorrendo sobre o uso do conhecimento tradicional no uso das plantas medicinais no Brasil para o tratamento da Diabetes Mellitus e os efeitos hipoglicemiantes frente à terapia medicamentosa. Pode-se concluir que os sujeitos adoecidos com Diabetes Mellitus contam com uma variedade de práticas terapêuticas com plantas medicinais para o tratamento, mas seus efeitos devem ser melhor estudados em seres humanos, de forma a assegurar o uso dessas plantas.
\end{abstract}

Palavras-chave: Prática terapêutica; Plantas medicinais; Diabetes Mellitus.

\begin{abstract}
The records of therapeutic practices with the use of medicinal plants range from the prevention, treatment and cure of diseases, being of great relevance to human history. Thus, this article aims to identify the medicinal plants used in therapeutic practice for the treatment of Diabetes Mellitus. This research is also justified due to the large number of people affected by the disease. Therefore, a bibliographical, exploratory and qualitative research was carried out. Thus, to compose this research, a search was performed in databases (Google Scholar), (PubMed) and (Scielo) using descriptors in Health Sciences (DeCS): diabetes mellitus" and "medicinal plants" or their equivalents in English and Spanish, being selected the references covering the period from 1993 to 2021. Discussing the use of traditional knowledge in the use of medicinal plants in Brazil for the treatment of Diabetes Mellitus and the hypoglycemic effects of drug therapy. It can be concluded that the subjects sick with Diabetes Mellitus have a variety of therapeutic practices with medicinal plants for their treatment, but their effects should be better studied in human beings, in order
\end{abstract}


to ensure the use of these plants.

Keywords: Therapeutic practice; Medicinal plants; Diabetes Mellitus.

\section{Resumen}

Los registros de prácticas terapéuticas con el uso de plantas medicinales van desde la prevención, tratamiento y curación de enfermedades, siendo de gran relevancia para la historia humana. Así, este artículo tiene como objetivo identificar las plantas medicinales utilizadas en la práctica terapéutica para el tratamiento de la Diabetes Mellitus. Esta investigación también se justifica por la gran cantidad de personas afectadas por la enfermedad. Por ello, se realizó una investigación bibliográfica, exploratoria y cualitativa. Así, para componer esta investigación se realizó una búsqueda en bases de datos (Google Scholar), (PubMed) y (Scielo) utilizando descriptores en Ciencias de la Salud (DeCS): diabetes mellitus "y" plantas medicinales "o sus equivalentes en inglés y español, siendo seleccionadas las referencias que cubren el período de 1993 a 2021. Discutir el uso de los conocimientos tradicionales en el uso de plantas medicinales en Brasil para el tratamiento de la Diabetes Mellitus y los efectos hipoglucemiantes de la farmacoterapia. Se puede concluir que los sujetos enfermos de Diabetes Mellitus tienen una variedad de prácticas terapéuticas con plantas medicinales para su tratamiento, pero conviene estudiar mejor sus efectos en el ser humano, a fin de asegurar el uso de estas plantas.

Palabras clave: Práctica terapéutica; Plantas medicinales; Diabetes Mellitus.

\section{Introdução}

O homem desde a antiguidade utiliza de recursos naturais disponíveis como opção de cura e tratamento de diversas enfermidades. Em razão da facilidade de acesso, o uso das plantas medicinais tem sido revelado em diferentes povos ao redor do globo, sendo adotada até os dias atuais com o objetivo de gerar melhoria na qualidade de vida das pessoas, assim como a manutenção da saúde (Castro \& et al., 2020).

O uso de plantas in natura ou plantas secas de forma orgânica e preparadas através do conhecimento e experiências empíricas caracteriza a fitoterapia tradicional (Rezende \& Cocco, 2002; Santos \& Rudzit, 2014). Sendo essa em alguns casos, seja pela distância dos grandes centros ou pelas condições socioeconômicas, a única opção viável ao tratamento de doenças dependendo assim das práticas tradicionais na atenção primária a saúde (Pantoja \& Lopes, 2013).

Das 200.000 espécies de plantas nativas do Brasil, estima-se que metade tenha alguma finalidade terapêutica e que apenas $1 \%$ dessas espécies com potencial foi alvo de estudos (Vieira \& et al., 2016). Nesse contexto, surge o seguinte problema de pesquisa: Como a prática terapêutica das plantas medicinais pode colaborar para o tratamento da Diabetes Mellitus?

O Diabetes Mellitus surge como uma desordem metabólica e estima-se que até 2025, 333 milhões de pessoas apresentarão diagnóstico positivo para diabetes mellitus, ou seja, cerca de 8\% da população adulta (Codogno \& et al., 2012). Dessa forma, justifica-se essa pesquisa também devido ao grande número de pessoas acometidas pela doença. Pessoas acometidas pela doença vêm utilizando plantas medicinais a partir de um conhecimento compartilhado ao longo das gerações, e que, muitas vezes, constitui o único recurso em termos de cuidados médicos, curativos ou preventivos (Paixão, 2016).

Este artigo tem como objetivo identificar as plantas medicinais utilizadas como prática terapêutica para o tratamento do Diabetes Mellitus.

\section{Metodologia}

Esse artigo foi desenvolvido a partir de uma pesquisa bibliográfica, tendo o caráter teórico, qualitativo e exploratório. A pesquisa bibliográfica para Mioto e Lima (2007), é um procedimento metodológico importante para o conhecimento científico. "A pesquisa bibliográfica é a que se desenvolve tentando explicar um problema, utilizando o conhecimento disponível a partir das teorias publicadas em livros ou obras congêneres" (Köche, 2011, p. 122). Juntamente com a pesquisa bibliográfica, a pesquisa exploratória se torna importante para o pesquisador aprofundar a temática (Pereira \& et al., 2018).

Na busca de material bibliográfico para dar suporte qualitativo à pesquisa foram identificadas algumas bases de dados: 
Google Scholar, PubMed e Scielo. Nessas bases de dados foram identificados artigos científicos redigidos nos idiomas Português, Inglês e Espanhol, com delineamento experimental (ensaios clínicos, randomizados ou não), observacionais (descritivos e analíticos) e de revisão (de literatura, sistemáticos e meta-análises) e dissertação de mestrado. Esse material bibliográfico identificada data do período de 1993 e 2021.

A análise qualitativa sobre o referencial teórico compôs a seção de resultados e discussão e permitiu aos autores verificar como as ideias propostas sobre a temática do uso das plantas medicinais para tratamento da Diabetes Mellitus vêm sendo estudadas.

Os principais Descritores em Ciências da Saúde (DeCS) utilizados na base: "diabetes mellitus" e "plantas medicinais", utilizadas separadamente ou em combinação, através do operador booleano "and" para as palavras-chave em inglês e "e" para português.

\section{Resultados e Discussão}

Nesta seção, será discutido inicialmente sobre o conhecimento e o uso de plantas medicinais. Em seguida, serão investigados outros dois termos como Diabetes Mellitus no Brasil e como é o uso de plantas medicinais no Diabetes Mellitus.

\subsection{Conhecimento tradicional e o uso de plantas medicinais}

O conhecimento tradicional, quando sustentado pela comprovação científica, pode estabelecer uma prática terapêutica segura e eficaz, assim como o uso de plantas medicinais e fitoterápicos em uma dimensão social e econômica (Matos, 2008). As plantas medicinais contêm mistura de diferentes metabólitos secundários, ou seja, um fito complexo que pode ter ação individual, aditivamente, ou em contribuição para o restabelecimento da saúde do indivíduo. A compreensão da ação de extratos vegetais no organismo, bem como o potencial farmacológico dos variados compostos químicos multifuncionais se faz importante no tratamento de diversas patologias (Rodrigues \& Barbano, 2007).

A criação do conhecimento existe quando se tem a análise de discussões referentes às diferenças de dados, informação e conhecimento. Há vários autores que buscam enfatizar essa diferença entre dados, informação e conhecimento (Dutta, 1997; Marshall, 1997; Davenport \& Prusak, 1998), porém sem um consenso da diferenciação entre esses três conceitos.

Dentro dessas definições e diferenciações hierárquicas, o conhecimento é formado por informações, que podem ser verbalizadas, exteriorizadas dentro de uma característica subjetiva instável e dinâmica, que se encontra na mente das pessoas, onde essas informações envolvem experiência, contexto, interpretação e reflexão (Nonaka \& Takeuchi, 1997).

Essa socialização, combinação, internalização e externalização do conhecimento é observada na história do ser humano desde a era paleolítica onde a história vem intrinsecamente ligada ao ambiente natural, especialmente as plantas, utilizadas para alimentação, confecção de moradia e utensílios, vestuários e remédios.

O uso dos vegetais para o tratamento de diversas doenças remonta ao início da civilização e ainda é utilizado em todo o mundo, seja nos grandes centros urbanos ou em comunidades rurais (Rossato, 2012). Segundo a OMS 1993, 80\% da população mundial recorre às medicinas tradicionais para atender suas necessidades primárias de assistência médica.

O Brasil possui uma das maiores biodiversidades ecológicas do planeta, assim como um precioso conhecimento tradicional quanto ao uso de plantas medicinais frente à diversidade étnica e cultural e mesmo tendo um potencial terapêutico muito grande, o caminho rumo a aceitação do uso das plantas como alternativa terapêutica ainda é longo no meio acadêmico, em virtude da pequena quantidade de estudos clínicos sobre os mecanismos de ação, efeitos e riscos no uso destas substâncias (Santos \& et al., 2012).

Diante do seu vasto patrimônio genético e ampla diversidade cultural, o Brasil tem a possibilidade de estabelecer um modelo de desenvolvimento próprio no Sistema Único de Saúde (SUS) para o uso de plantas medicinais e fitoterápicas. Esse 
modelo garante uma maior universalização do acesso a essa alternativa terapêutica, visando a sustentabilidade econômica e ecológica, respeitando princípios éticos e ainda proporcionando a inclusão social (Panizza, 2010).

As plantas medicinais e os fitoterápicos estão entre os principais recursos terapêuticos da Medicina Complementar e Alternativa e vêm sendo utilizados há muito tempo pela população brasileira em seus cuidados com a saúde na Medicina Tradicional/Popular ou ainda nos programas de fitoterapia no âmbito do SUS, alguns ultrapassam 20 anos de existência. Entre as Práticas Integrativas e Complementares no SUS, as plantas medicinais e a fitoterapia são as mais presentes no sistema, segundo o diagnóstico do Ministério da Saúde, onde a maior parte das experiências acontecem na Atenção Primária à Saúde (Nascimento, 2016).

O uso das plantas medicinais passou a ser um recurso importante dos profissionais de saúde, usuários e gestores. O Ministério da Saúde criou a Política Nacional de Práticas Integrativas e Complementares, além de criar a Política Nacional de Plantas Medicinais e Fitoterápicos e o Programa Nacional de Plantas Medicinais e Fitoterápicos. Em 2008, foi publicada a Relação Nacional de Plantas Medicinais de Interesse do SUS (RENISUS) abrangendo 71 plantas (Brasil, 2009).

Além das políticas públicas criadas pelo Ministério da Saúde, há ainda a RDC n 26/2014 que divide os produtos derivados de plantas em duas categorias, sendo a primeira sobre os medicamentos fitoterápicos que devem apresentar segurança e eficácia através de ensaios clínicos e, por segundo, os produtos tradicionais fitoterápicos que poderão ser registrados através da comprovação de tradicionalidade (Brasil, 2014).

Um estudo realizado na região de Colombo no Paraná avaliou o conhecimento sobre plantas medicinais por usuários da Unidade Básica de Saúde e, nos dados encontrados por eles, observou-se que a prevalência de utilização de plantas medicinais $(72,28 \%)$ foi por indivíduos do sexo feminino, com idade inferior a 40 anos, baixa renda e escolaridade (Oliveira \& et al., 2018).

Outro estudo realizado com moradores do bairro do sossego no distrito de Marudá - PA, buscou avaliar a sabedoria popular no uso de plantas medicinais e realizou uma comparação entre o número de plantas citadas e a idade dos entrevistados e verificou-se que as mulheres entre 64 e 87 anos apresentaram maior número de citações sobre o uso, constatando que, de maneira geral, os mais idosos conhecem uma maior diversidade de plantas utilizáveis, por causa do saber acumulado ao longo de suas vidas (Flor \& Barbosa, 2015).

De acordo com Cavaglier (2014), mesmo com o avanço da medicina em diversas partes do mundo, no Brasil, as plantas medicinais costumam ser uma escolha para parte da população, principalmente a de baixa renda, por diversos fatores, dentre eles o custo alto dos medicamentos industrializados e a dificuldade de acesso a um sistema de saúde eficiente. Por outro lado, o consumo desse tipo de terapia tem crescido também entre as pessoas de maior condição socioeconômica, na busca por opções terapêuticas mais saudáveis.

A cultura e o cultivo das plantas medicinais, principalmente em comunidades interioranas, representam um importante recurso local para saúde e sustentabilidade do meio ambiente rural. No entanto, a orientação quanto ao manejo e uso correto se faz importante, pois a complementação do conhecimento popular e científico sobre a produção e uso correto das plantas medicinais no Brasil é fundamental para maior eficácia e segurança dos seus usuários (Flor \& Barbosa 2015).

Estudos realizados Figueiredo e Filho (2015), mostram que derivados de plantas medicinais com ação hipoglicemiante estão sendo usadas tanto na medicina popular, quanto nos sistemas tradicionais de saúde em todo mundo, bem como muitos produtos farmacológicos modernos são derivados de plantas, como a Metformina (hipoglicemiante oral) que é um medicamento utilizado no tratamento da Diabetes Mellitus e é derivada da Galega officinales.

Propostas que proporcionem o embasamento técnico aos profissionais quanto ao uso das plantas medicinais podem permitir um diálogo fundamentado e produtor de conhecimento e dessa forma, a ampliação do seu uso como prática instituída nos serviços de saúde, que podem ser definidos como modos de saber fazer atos de saúde que operem sobre as necessidades, 
uma vez que os envolvidos, sejam eles profissionais ou usuários, validam o conhecimento dos saberes tecnológicos que agem no campo da saúde (Alcântara \& et al., 2015).

\subsection{Diabetes Mellitus no Brasil}

As doenças crônicas não transmissíveis (DCNTs) foram responsáveis por 73\% das mortes gerais e $17 \%$ das mortes precoces registradas no ano de 2017 no Brasil (Who, 2017). O Diabetes Mellitus (DM) é um crescente e relevante problema de saúde para todos os países, independentemente do seu grau de desenvolvimento e no Brasil não é diferente. Em 2019 no Brasil houve cerca de 16,8 milhões de pessoas com diabetes mellitus no país, sendo o quinto no mundo com pessoas entre 20-79 anos diagnosticadas com a doença segundo a Federação Internacional de Diabetes (IDF).

Para o tratamento apropriado e definição de estratégias de rastreamento e prevenção de complicações ocorridas pelo Diabetes Mellitus a sua classificação é fundamental, pois de acordo com a Sociedade Brasileira do Diabetes (SBD,2021) sua classificação deve ser baseada na etiopatogenia do diabetes, que abrange o diabetes tipo 1 (DM1), o diabetes tipo 2 (DM2), o diabetes gestacional (DMG) e os outros tipos de diabetes. Há ainda outras classificações que têm sido propostas de acordo com os subtipos de Diabetes Mellitus considerando as características clínicas, tais como o início dos sintomas, a história familiar, a função residual das células beta, os índices de resistência à insulina, risco de desordens crônicas, grau de obesidade, a presença de autoanticorpos e possíveis características sindrômicas (SBD, 2021).

O Diabetes Mellitus tipo 2 (DM2) equivale a 90 a 95\% de todos os casos de diabetes mellitus. É de etiologia multifatorial e complexa que envolve fatores genéticos e ambientais. Normalmente acomete indivíduos a partir da quarta década de vida, apesar de ser descrito o aumento da sua incidência em crianças e jovens. Trata-se de uma doença poligênica, com fatores genéticos fortemente envolvidos, ainda que não completamente esclarecidos, cuja ocorrência tem contribuição de fatores ambientais, dentre eles hábitos dietéticos e sedentarismo que contribuem para obesidade e destacam-se como os principais fatores de risco. Em grande parte dos casos, a doença é assintomática ou oligossintomática por um longo período de tempo, sendo necessário realizar o diagnóstico por dosagens laboratoriais de rotina ou a partir de manifestações das complicações crônicas (Sociedade Brasileira de Diabetes, 2019).

Quando ocorre a descompensação do diabetes muitas consequências podem ocorrer, inclusive elas podem resultar em morte prematura do sujeito acometido pela enfermidade. As complicações podem ser divididas em agudas e crônicas, sendo as consequências agudas mais comuns cetoacidose diabética, o coma diabético, infecções e perda de consciência. As complicações crônicas se dividem em micro e macro vasculares, sendo as micro vasculares a nefropatia, retinopatia e neuropatia diabética. Já as complicações macro vasculares fazem parte a encefalopatia diabética, doença arterial coronariana, doença arterial periférica e o pé diabético (IDF, 2017).

O Diabetes Mellitus atinge em todo o mundo grande número de pessoas de qualquer condição social, tornando-se problema de saúde pública de grande relevância epidemiológica, visto que a sua incidência e prevalência têm aumentado nos últimos anos e já vêm alcançando proporções epidêmicas. O manejo deve ser realizado dentro de um sistema de saúde hierarquizado, sendo sua base o nível primário. Na prestação de serviços a esses sujeitos é necessário serem considerados os principais componentes do sistema de saúde, especialmente a determinação das necessidades e dos recursos locais (Assunção \& et al., 2001).

Dados referentes à Pesquisa Nacional de Saúde, realizada em 2014, apontam que 47\% dos usuários com diagnóstico de Diabetes Mellitus referem ter recebido assistência médica nos últimos 12 meses nas Unidades Básicas de Saúde (UBSs), e 29\% em consultórios ou clínicas privadas. Esta mesma pesquisa aponta ainda que 5\% dos usuários com diagnóstico de Diabetes Mellitus há menos de dez anos e 5,8\% com diagnóstico há mais de dez anos apresentam feridas nos pés. A amputação de membros acontece em 0,7 e 2,4\% desses, respectivamente, o que demonstra ser um percentual bastante significativo, sendo 
a amputação uma complicação irreversível com implicações físicas, mentais e sociais extremas (Brasil, 2014).

O estabelecimento do diagnóstico do Diabetes Mellitus deve ser realizado através do reconhecimento da hiperglicemia. Para que assim seja feito os testes indicados são a glicemia plasmática de jejum, teste oral de tolerância à glicose (TOTG) e a hemoglobina glicada (A1c). Em algumas circunstâncias é recomendado a realização do rastreamento de pacientes assintomáticos (SBD, 2021).

O aumento do número de sujeitos com diagnóstico de doenças crônicas e também do emprego das plantas medicinais por esses, reforça a necessidade em conhecer o uso dessa prática terapêutica, de forma a respaldar a formulação de novas políticas públicas, reforçar as que já existem no âmbito do SUS e melhorar a atuação dos profissionais de saúde na área. A relevância em se aprofundar nesse conhecimento decorre da demanda pelo fortalecimento de evidências sobre a atuação terapêutica das plantas, a produção de efeitos colaterais e interações com a medicina alopática. Somado a isso, o conhecimento gerado poderá relativizar o paradigma positivista em saúde, valorizar o conhecimento tradicional, o uso sustentável da biodiversidade brasileira, além do fortalecimento da agricultura familiar (Siqueira \& et al., 2017).

\subsection{O uso de plantas medicinais para o tratamento de Diabetes Mellitus}

Durante séculos, muitas plantas foram consideradas uma fonte fundamental de medicamentos antidiabéticos potentes. Em países em desenvolvimento, particularmente, as plantas medicinais são usadas no tratamento do diabete mellitus para superar o alto custo dos medicamentos convencionais para a população. Hoje em dia, os tratamentos de doenças, incluindo diabetes, usando plantas medicinais são recomendados porque essas plantas contêm vários fitoconstituintes, como flavonóides, terpenóides, saponinas, carotenóides, alcalóides e glicosídeos, que podem possuir atividades antidiabéticas (Kooti \& et al., 2015).

De acordo com o Ministério da Saúde 2012, são consideradas plantas medicinais todas as plantas frescas (in natura) que são coletadas no momento do uso, as plantas secas que, após coletadas, são estabilizadas que passem pelo processo de secagem (permanecendo íntegras, rasuradas, trituradas ou pulverizadas) e que podem ser usadas como chás caseiros e preparadas de modo artesanal no domicílio.

O Brasil possui uma das maiores biodiversidades ecológicas do planeta, assim como um precioso conhecimento tradicional quanto ao uso de plantas medicinais frente à diversidade étnica e cultural. Neste contexto, mesmo tendo um potencial terapêutico muito grande, o caminho rumo a aceitação do uso das plantas como alternativa terapêutica ainda é longo no meio acadêmico, em virtude da pequena quantidade de estudos clínicos sobre os mecanismos de ação, efeitos e riscos no uso destas substâncias (Santos \& et al., 2012).

As plantas medicinais representam a origem de $25 \%$ dos fármacos utilizados, fato esse que se deve à extensa flora mundial e as importantes propriedades terapêuticas destas plantas. No Brasil, diversas pesquisas vêm sendo realizadas contribuindo substancialmente para o desenvolvimento e o uso destas espécies vegetais, uma vez que seus efeitos terapêuticos vêm sendo comprovados, o que possibilita a utilização destas no tratamento de doenças (Kalluf, 2008).

$\mathrm{Na}$ atualidade, há um grande conjunto de fármacos adotados para a melhoria dos índices glicêmicos em sujeitos com diagnóstico de Diabetes Mellitus, tais como a insulina e os hipoglicemiantes orais. Entretanto, mesmo quando as intervenções medicamentosas demonstram efeitos positivos, o alto custo e a quantidade de efeitos colaterais destas drogas têm provocado o interesse de pesquisadores sobre o conhecimento de substâncias naturais na redução dos níveis glicêmicos de sujeitos com diagnóstico de Diabetes Mellitus, visto que muitos destes optam pela suplementação dietética e de terapias alternativas como o uso das plantas medicinais (Brito \& et al., 2020).

Vale ressaltar que o uso das plantas medicinais tem uma maior adesão entre os idosos e que é mais comum este emprego das plantas de forma associada à terapia medicamentosa (Brito \&, 2020) Dentre as plantas usadas pelo público com 
diabetes mellitus, algumas já constam na Relação Nacional de Plantas Medicinais de Interesse ao SUS (Renisus), tais como: Allium Sativum Liliaeceae (alho), Bauhinia Forficata (pata-de-vaca), Matricaria Chamomilla (camomila), Ocimum. Gratissimum (alfavaca) e Passiflora Alata Curtis (maracujá) (Brasil, 2009).

O Allium sativum $L$. (alho) apresenta compostos bioativos como flavonóides (Souza, 2017), tal ativo tem a finalidade terapêutica que vem ao encontro do que consta no Renisus, do Ministério da Saúde, que associa o mesmo com a redução das pressões sistólicas e diastólicas, inibição da enzima conversora (ECA), assim como efeitos antioxidantes, cardioprotetor, antiinflamatório, diurético, hipolipidêmico, imune- estimulante e antiplaquetário (Brasil, 2015).

Dentre os vários nutrientes encontrados com efeitos terapêuticos no alho foi encontrado proteínas, ácidos graxos, carboidratos e vitaminas como a A, $\mathrm{B}_{1}, \mathrm{~B}_{2}$ e C (Apolinario \& et al., 2008). E em um dente de alho foi identificado também cerca de 33 compostos organossulfurados como a Liina, Ajoeno, Alicina, Tiosulfato, Alil-mercaptnao, S-acil-cisteína e compostos gama-glutâmicos (Cardoso \& Nepomuceno, 2015). A alicina sobresai sobre os demais compostos organossulfurados, devido a sua capacidade de dimnuir o nível glicêmico, podendo ser comparada a ação da insulina. O mecanismo de ação ainda não se sabe ao certo, podendo agir na elevação pancreática de insulina, estimulando as células $\beta$ na sua produção ou regeneração das mesmas (Oliveira \& et al., 2018).

A pata-de-vaca (Bauhinia forficata) é uma das plantas de grande utilização pela população para o controle do Diabetes Mellitus. Seus efeitos hipoglicemiantes decorrem, em grande parte, do extrato aquoso de suas folhas e raízes. Estudos comparativos realizados com camundongos normoglicêmicos e hiperglicêmicos aplicando o extrato aquoso da pata-de-vaca demostraram que seus constituintes químicos, tais como a canferitrina, canferol e flavonoides tiveram resultados promissores para a ação hipoglicemiante. Seu mecanismo de ação pode ser atribuído ao seu potencial de inibição da enzima catalisadora dos açúcares, a partir da atividade dos constituintes químicos quercetina e canferol que promovem a redução da glicemia no sangue (Pontes \& et al., 2017).

As folhas desta espécie são comumente utilizadas como chás, uma vez que, em muitas comunidades rurais esta planta é conhecida como pata-de-vaca verdadeira (Silva \& Cechinel-Filho, 2002). A utilização desta espécie como alternativa terapêutica de Diabetes Mellitus, vem vendo amplamente relatada na literatura, como verificado por Trojan-Rodrigues (2012) em uma análise de estudos etnobotânicos em que a Bauhinia Forficata teve um maior destaque entre as plantas relatadas em seu uso popular para tratar Diabetes Mellitus no Estado do Rio Grande do Sul, além de ser uma espécie que é largamente comercializada no Brasil.

A Aloe Vera ou popularmente conhecida como babosa, tem sido muito utilizada devido as suas inúmeras propriedades terapêuticas, dentre elas, conhecidamente, a atividade hipoglicemiante. No estudo feito por Negri (2005) esse efeito foi validado em ratos que possuíam diabetes tipo 1 e 2 quando em contato com seu extrato aquoso, realizado a partir de suas folhas. A pesquisa demostrou, inclusive, um maior efeito antidiabético nos ratos com diabetes tipo 2 do que o medicamento glibencamida, amplamente utilizado para o tratamento da doença.

Em 2021 um estudo para compreensão do mecanismo de ação da Aloe vera (carboidratos e polipeptídeos) no alívio da Diabetes foi feito e demonstrou que os carboidratos aliviam o diabetes por meio de captação de glicose e um mecanismo antioxidante, e a fração polipeptídica atua por meio da restauração da permeabilidade intestinal pela redução dos níveis de zonulina, assim o efeito sinérgico desses consttituintes leva a Aloe vera como uma planta com grande potencial para o alivio do diabetes e de sua progressão (Babu \& et al., 2021). Comparando com a metformina a aloe vera apresenta uma maior eficácia como agente anti-hiperglicêmico, podendo ser usado como tratamento de primeira linha para a Diabetes Mellitus, seguido de metformina, tendo sempre o cuidado de fazer o uso em curto período da Aloe vera devido aos seus efeitos adversos (Rehman $\&$ et al., 2020).

No estudo realizado por Parestrelo (2018) em que foi investigado o potencial antioxidante do chá de camomila nas 
glândulas salivares e sua influência no estado glicêmico de ratos diabéticos confirmou seu potencial hipoglicemiante, bem como a ação do chá no controle da polidipsia, sintoma comum em pacientes com Diabetes Mellitus. Os resultados obtidos confirmaram o potencial antioxidante do chá na glândula parótida dos animais diabéticos após o tratamento, concluindo que o chá de camomila é apontado como promissor na prevenção dos danos oxidativos presentes no Diabetes Mellitus, tanto nas glândulas salivares como na redução do malondialdeído (MDA, um marcador para o estresse oxidativo) quanto pelo seu potencial hipoglicemiante.

Uma pesquisa realizada por Pivari et al. (2019) buscou compreender os mecanismos moleculares e celulares já descobertos em modelos in vivo e in vitro da curcumina para o tratamento da Diabetes Mellitus 2. Os estudos demonstraram que a curcumina melhora os eventos patológicos na Diabetes Melittus 2 por meio de diferentes mecanismos e múltiplos alvos moleculares. Em particular, a curcumina está envolvida na regulação do metabolismo lipídico. A curcumina reduz a expressão gênica de fatores de transcrição envolvidos na lipogênese hepática, além disso foi comprovado que a curcumina aumenta a atividade das enzimas de mobilização lipídica. Os dados relatados mostram que a curcumina tem potencial terapêutico para neutralizar o diabetes e suas complicações, uma vez que todos os estudos descritos na pesquisa demonstraram que doses de até 12 g por dia de curcumina são seguras, toleráveis e não tóxicas.

Diversos mecanismos são descritos, explicando os efeitos benéficos dos fitoquímicos, como regulação do metabolismo de glicose e lipídios, secreção de insulina, células $\beta$ estimulantes, via de sinalização de NF-kB, inibição de enzimas gliconeogênicas e ação protetora de ROS. Nessa relação, a investigação dos fitoquímicos responsáveis pelos efeitos antidiabéticos tem progredido nas últimas décadas. O tratamento da Diabetes Mellitus com compostos derivados de plantas, que são acessíveis e não requerem síntese farmacêutica laboriosa, parece altamente atraente (Pivari \& et al., 2019).

\section{Considerações Finais}

Ao longo dessa pesquisa foi possível identificar que o uso de plantas com a finalidade terapêutica não é uma prática recente, sendo observada desde a antiguidade. Essa prática realizada por diversos povos pode colaborar na manutenção da saúde.

Apesar de não haver comprovação científica elucidada sobre o efeito hipoglicemiante para todas as plantas medicinais registradas com este fim, os estudos sugerem que o mecanismo de ação antidiabético pode ser exemplificado por seu potencial estimulador de células beta pancreáticas, que contribuem ao aumento da liberação do hormônio insulina; por sua capacidade de subir o consumo de glicose pelos tecidos e órgãos; ou ainda, por aumentar o número e a sensibilidade dos receptores de insulina.

Em resposta ao problema de pesquisa - Como a prática terapêutica das plantas medicinais pode colaborar para o tratamento da Diabetes Mellitus? -, destacam-se as seguintes considerações:

- A prática terapêutica com uso das plantas medicinais em seres humanos para o tratamento da Diabetes Mellitus deve ser fortalecida por pesquisas que comprovem a eficácia dos fitoconstituintes presentes nas plantas;

- Essas pesquisas podem contribuir também para identificar o perfil de toxicidade no uso dessas plantas a longo prazo em sujeitos adoecidos com Diabetes Mellitus, com a acompanhamento da evolução das complicações tais como a neuropatia diabética e nos fenômenos micro e macro vasculares;

- Ademais, esses estudos podem estimular o uso tradicional destas espécies e diminuir a exposição às práticas pouco seguras.

Pode-se concluir que os sujeitos adoecidos com Diabetes Mellitus contam com uma variedade de práticas terapêuticas com plantas medicinais para o tratamento, mas seus efeitos devem ser melhor estudados em seres humanos, de forma a assegurar o uso dessas plantas. 
Sugestões para pesquisas futuras: estudos detalhados sobre a fitoquimíca de cada planta medicinal já utilizada de modo empírico no tratamento da Diabetes Mellitus; estudos envolvendo as separações dos compostos ativos dessas plantas; e , pesquisas sobre a dosagem e nível de toxidade dos compostos ativos na fisiologia no corpo humano, levando em consideração todas as condutas éticas pertinentes para pesquisas com seres humanos.

\section{Referências}

Alcântara, L. G. R., Joaquim, T. V. H. \& Sampaio, F. S. (2015). Plantas medicinais: Conhecimento e Uso Popular. Revista de APS. https://periodicos.ufjf.br/index.php/aps/article/view/15680/8212

Apolinario, A. C., Monteiro, M. M. O., Pachú, C. O. \& Dantas, I. C. (2008) Allium sativum L. Como agente terapêutico para diversas Patologias: uma revisão. BioFar Revista de biologia e farmácia. 3(1),:https://scholar.google.com/scholar?as_ylo=2017\&q=Allium+sativum+L.+e+diabetis+mellitus\&hl=ptBR\&as_sdt $=0,5$

Almeida, M. Z. (2011). Plantas Medicinais. (3a ed.), EDUFBA, http://books.scielo.org/id/xf7vy/pdf/almeida-9788523212162-03.pdf

Assunção, M. C. F., Santos, I. S. \& Gigante, D. P. (2001). Atenção primária em diabetes no sul do Brasil: estrutura, processo e resultado. Revista Saúde Pública, São Paulo, (35)1, 88-95. https://www.scielosp.org/article/rsp/2001.v35n1/88-95/pt/

Babu, S. N., Govindarajan, S. \& Noor, A. (2021). Aloe vera and its two bioactive constituents in alleviation of diabetes-proteomic \& mechanistic insights. Journal of Ethnopharmacology, 114445. https://doi.org/10.1016/j.jep.2021.114445

Barbosa, L. C. S. (2019). Plantas Medicinais no Tratamento Suplementar de Diabetes Mellitus Presente na Renisus: Uma Revisão de Literatura. VII Simpósio de Plantas Medicinais do Vale do São Francisco - PLAMEVASF. http://www.plamevasf.univasf.edu.br/arquivos_anais/Fit2727.pdf

Beber, B., Silva, E. \& Bonfiglio, U. (2014). Metacognição como processo de aprendizagem. Revista Psicopedagogia, (3)95, http://revistapsicopedagogia.com.br/detalhes/74/metacognicao-como-processo-da-aprendizagem.

Brasil. (2009). Ministério da Saúde. Secretaria de Ciência, Tecnologia e Insumos Estratégicos. Departamento de Assistência Farmacêutica e Insumos Estratégicos. Programa Nacional de Plantas Medicinais e Fitoterápicos / Ministério da Saúde, Secretaria de Ciência, Tecnologia e Insumos Estratégicos, Departamento de Assistência Farmacêutica e Insumos Estratégicos. Brasília, 43-49. https://bvsms.saude.gov.br/bvs/publicacoes/progr ama_nacional_plantas_medicinais_fitoterapicos.pdf

Brasil. (2012). Ministério da Saúde. Práticas integrativas e complementares: plantas medicinais e fitoterapia na Atenção Básica.

Brasil. (2014). Ministério da Saúde. Agência Nacional de Vigilância Sanitária. Resolução da Diretoria Colegiada - RDC n²6. https://bvsms.saude.gov.br/bvs/saudelegis/anvisa/2014/rdc0026_13_05_2014.pdf

Brito, V. P. Freitas, M. C. F. Gomes, D. C \& Oliveira, V. O. (2020). A fitoterapia como uma alternativa terapêutica complementar para pacientes com diabetes mellitus no Brasil: Uma revisão sistemática. Revista Saúde Meio Ambiente, (9), 189-204. https://doi.org/10.24302/sma.v9i0.2847

Cardoso, A. C. M. \& Nepomuceno, J. C. (2015). Avaliação do efeito modulador do óleo de alho (Allium Sativum L.) sobre a carcinogenicidade da doxorrubicina em células somáticas de Drosophila melanogaster. Revista Perquirere, (12)1, 160-175, https://scholar.google.com/scholar?as_ylo=2017\&q=Allium+sativum+L.+e+diabetis+mellitus\&hl=pt-BR\&as_sdt=0,5

Castro, I. B \& et al., (2020). Estratégias nutricionais no tratamento do diabetes mellitus: revisão bibliográfica. Revista Reseach, Society and Development, (9)2, http://dx.doi.org/10.33448/rsd

Cavaglier, M. C. S. \& Messeder, J. C. (2014). Plantas Medicinais no Ensino de Química e Biologia: Propostas Interdisciplinares na Educação de Jovens e Adultos. Revista Brasileira de Pesquisa em Educação em Ciências. Minas Gerais. (p.12). https://www.semanticscholar.org/paper/Plantas-Medicinais-noEnsino-de-Qu\%C3\%ADmica-e-Biologia\%3A-Cavaglier-Messeder/d5ed4bc8d2adf550d0a17a51f2043c8e1b2bc76

Codogno, J. S., Fernandes, R. A. \& Monteiro, H. L. (2012). Prática de atividades físicas e custo do tratamento ambulatorial de diabéticos tipo 2 atendidos em unidade básica de saúde. Arquivos Brasileiros de Endocrinologia \& Metabologia, 56(1), 6-11, https://doi.org/10.1590/S0004-27302012000100002

Consenso Brasileiro sobre Diabetes. (2000). Diagnóstico e classificação do Diabetes Mellitus e tratamento do diabetes mellitus tipo 2 - Versão final e definitiva. Recomendações da Sociedade Brasileira de Diabetes. Retirado de https://bvsms.saude.gov.br/bvs/publicacoes/consenso_bras_diabetes.pdf

Davenport, T. H. \& Prusak, L. (1998). Conhecimento empresarial. https://www.scirp.org/reference/References Papers.aspx?ReferenceID=1664783

De Pontes, M. A. N. \& et al., (2017). Bauhinia forficata L. e sua a ação hipoglicemiante. Archives of health investigation, (6)11. https://doi.org/10.21270/archi.v6i11.2244

Dutta, S. (1997). Strategies for implementing knowledge-based systems. IEEE Transactions on Engineering Management (44)1. 79-99. https://web.archive.org/web/20040423024548/http://www.mgt.ncu.edu.tw:80/ choulab/km2004/IEEE/Strategies_for_Implement_Knowledge-

Based_Syatems.pdf

Figueiredo, C. A., Gurgel, I. G. D \& Junior, G. D. (2014). A Política Nacional de Plantas Medicinais e Fitoterápicos: construção, perspectivas e desafios. Physis: Revista de Saúde Coletiva.São Paulo. https://doi.org/10.1590/S0103-73312014000200004

Flor. S. S. \& Barbosa, W. L. R (2015). Sabedoria popular no uso de plantas medicinais pelos moradores do bairro do sossego no distrito de Marudá. Revista Brasileira de Plantas Medicinais. 17, 113-119. https://www.scielo.br/j/rbpm/a/5X6gZSNb7rjgd7mLWQsrTZm/abstract/?lang=pt 
Kalluf, L. J. H. (2008). Fitoterapia Funcional: dos princípios ativos à prescrição de fitoterápicos. VP Editora. p. 304. https://pesquisa.bvsalud.org/portal/resource/pt/lil-558217

Karina, L. S. \& Valdir, C. F. (2002). Plantas do gênero Bauhinia: composição química e potencial farmacológico. Química Nova. 10.1590/S010040422002000300018

Köche, J. C. (2011). Fundamentos de metodologia científica: teoria da ciência e iniciação à pesquisa. Vozes.

Kooti, W. \& et al. (2015). Therapeutic and pharmacological potential of Foeniculum vulgare Mill: A review. Journal Herb Med Pharm, (4), 1-9. http://www.dmpjournal.org/article/320/10.11648.j.jdmp.20190504.11

Lakatos, E. M. \& Marconi, M. A. (2010). Fundamentos de metodologia científica. (7a ed.), Atlas.

Lima, M. (2007). Procedimentos metodológicos na construção do conhecimento científico: a pesquisa bibliográfica. Revista Katál (10), 37-45.

Lorenzi, H. \& Matos, F. J. A. (2008). Plantas medicinais no Brasil: nativas e exóticas. (2a ed.), Instituto Plantarum,544 p. http://www.agencia.cnptia.embrapa.br/gestor/bioma_caatinga/catalogo/REC000gbpasrvf02wx5ok07shnq94etlj3h.html

Matos, F. J. A. (2008). Plantas medicinais: guia de seleção e emprego de plantas usadas em fitoterapia no nordeste do Brasil. (3a ed.), Ed. UFC, p.06. Retirado de https://revistafitos.far.fiocruz.br/index.php/revista-fitos/article/view/85

Nascimento Júnior, B. J. \& et al., (2016). Avaliação do conhecimento e percepção dos profissionais da estratégia de saúde da família sobre o uso de plantas medicinais e fitoterapia em Petrolina-PE, Brasil. Revista Brasileira de Plantas Medicinais. Campinas, (18)1, https://doi.org/10.1590/1983-084X/15_031

Negri, G. (2005). Diabetes melito: plantas e princípios ativos naturais hipoglicemiantes. Revista Brasileira de Ciências Farmacêuticas, Brazilian Journal of Pharmaceutical Sciences, (41) 2, https://doi.org/10.1590/S1516-93322005000200002.

Nonaka, I. \& Takeuchi, H. (1997). Criação de conhecimento na empresa. Campus. https://books.google.com.br/books?id=FN_LCwX0s-

Oliveira, V. B. Mezzomo, T. R. \& Moaes. (2018). E.F.Conhecimento e Uso de Plantas Medicinais por Usuários de Unidades Básicas de Saúde na Região de Colombo, PR. Revista Brasileira de Ciências em Saúde, (22), 7, https://doi.org/10.22478/ufpb.2317-6032.2018v22n1.30038

Oliveira de, A. F. \& et al. (2018). Efeito hipoglicemiante do alho (Allium sativum L.) no diabético. VI Encontro De Monitoria E Iniciação Científica, XIV semana acadêmica. . https://scholar.google.com/scholar?as_ylo=2017\&q=Allium+sativum+L.+e+diabetis+mellitus\&hl=pt-BR\&as_sdt=0,5

Organização Mundial da Saúde. (1993). Unión Mundial Para La Natureleza (Uicn), World Wildlife Fund (WWF). Diretrizes sobre conservación de plantas medicinales. Londres. Retirado de https://www.urosario.edu.co/urosario_files/57/571bf298-6ad8-4b7f-b432-26a6fb78e6de.pdf

Panizza, S. T. (2010). Como prescrever ou recomendar plantas medicinais e fitoterápicos. COMBRAFITO. http://www.crfsp.org.br/images/cartilhas/Pla ntasMedicinais.pdf

Parestelo, B. O. (2018). Potencial antioxidante do chá de camomila nas glândulas salivares e sua influência no estado glicêmico de ratos diabéticos. Dissertação de Mestrado, Faculdade de Odontologia, Universidade de São Paulo, 10.11606/D.23.2018.tde-055112018-1225658

Pantoja, S. C. S \& Lopes, G. F. G. (2013). Levantamento das espécies de plantas medicinais utilizadas pela população de Santa Cruz, Revista Eletrônica Novo Enfoque, 16(16), 62-80. http://www.castelobranco.br/sistema/novoenfoque/files/16/6_SoniaPantoja140613_VF.pdf

Pereira A. S. \& et al. (2018). Metodologia da pesquisa científica. UFSM

Pivari, F. Mingione, A. Brasacchio, C. \& Soldati, L. (2019). Curcumin and Type 2 Diabetes Mellitus: Prevention and Treatment. Nutrients, $11(8), 1837$. $10.3390 /$ nu1 1081837

Rehman, M. T. \& et al. (2020). 44. Effect of Aloe vera and metformin on diabetic albino rats. Pure And Applied Biology (PAB), 9(3), 21222127. https://mail.thepab.org/index.php/journal/article/view/1464

Rezende, H. A \& Cocco, M. I. M. (2002) A utilização de fitoterapia no cotidiano de uma população rural, Revista Escola Enfermagem, USP, 36(3), 282-288. http://www.scielo.br/pdf/reeusp/v36n3/v36n3a10

Rodrigues, A. G. \& Barbano, D. B. A. (2007). Medicina tradicional/medicina complementar e alternativa no Sistema Único de Saúde: plantas medicinais e fitoterapia. https://bvsms.saude.gov.br/bvs/publicacoes/politica_nacional_fitoterapicos.pdf

Rossato, A. E. \& Chaves, T. R. C. (2012). Fitoterapia racional: aspectos taxonômicos, agroecológicos, etnobotânicos e terapêuticos, DIOESC, (1), 32-45. http://repositorio.unesc.net/bitstream/1/1628/2/Fitoterapia\%20Racional.pdf

Santos, M. M., Nunes, M.G.S. \& Martins, R. D. (2012). Uso empírico de plantas medicinais para tratamento de diabetes. Revista brasileira de plantas medicinais, (14) 2, 327-334. https://www.scielo.br/j/rbpm/a/MbK8PNkznz9Gvp4WqXfj5ny/?format=pdf\&lang=pt

Santos, A. M. C \& Rudzit, C. M. C. (2014). Uso de plantas medicinais por comunidades tradicionais costeiras de Cambury-Ubatuba-SP. In: Pereira, S. D. \& et al., Formação e Ocupação de litorais nas margens do atlântico -Brasil/Portugal, Editora Corbã, 101-109.

Trojan-Rodrigues, M. \& et al. (2012). Plants used as antidiabetics in popular medicine in Rio Grande do Sul. Journal Ethnopharmacol. 10.1016/j.jep.2011.10.034

Silva, F. A. (2017). Tratamento do diabetes mellitus tipo 2 através do uso de plantas medicinais. 2017. 41 f. Trabalho de Conclusão de Curso (Tese), Curso de Farmácia, Faculdade de Educação e Meio Ambiente - FAEMA, https://doi.org/10.31072/rcf.v9iedesp.616 
Research, Society and Development, v. 10, n. 10, e474101018874, 2021

(CC BY 4.0) | ISSN 2525-3409 | DOI: http://dx.doi.org/10.33448/rsd-v10i10.18874

Siqueira, J. B. V. \& et al. (2017). Uso de plantas medicinais por hipertensos e diabéticos de uma Estratégia de Saúde da Família Rural. Revista Contabilidade em Saúde. 12-17. https://doi.org/10.21527/2176-7114.2017.32.33-45

Sociedade Brasileira de Diabetes. (2019). Diretrizes Sociedade Brasileira do Diabetes 2019-2020. Editora Científica Clannad. https://www.diabetes.org.br/profissionais/images/DIRETRIZES-COMPLETA-2019-2020.pdf

Sociedade Brasileira de Diabetes. (2021). Diretriz 2021. https://diretriz.diabetes.org.br/classificacao-do-diabetes/

Vieira, A. C. M. \& et al. (2016). Manual sobre uso racional de plantas medicinais. Cerceav. https://books.googleuserconten t.com/books/ content?req=AKW5QaftybsbXyY60ZgkPO-VgVCgGGlihyPuHLsS9gEe3CSsys iCutptBemIwbhHZKzptUPYtn35IAC2XWecAS7xhmLm2K99B1_ aTapV oDTA i3sKZafc05pYUO-S0JfIxt7ARLUVAVK0K1z22zO8Cre42fIWSYJGTNOv8bgvAQ1 wbu-cv_9BJThmdn05yLgc7UyXS-Rvja_W_5L5MtzCpJ0 6b77OY vm eluWwuFww1G4I1mn2CFGC0hUjY4TBEJicu5KgTblieXboZsB4NdIIDFuptYS8giJ7prlN6KRmFvkkRC9rI5dq3Bk 